\title{
RESEARCH HIGHLIGHT The insulin receptor goes nuclear
}

\author{
Thiago M. Batista ${ }^{1}$, Carly T. Cederquist ${ }^{1}$ and C. Ronald Kahn ${ }^{1}$ \\ Cell Research (2019) 29:509-511; https://doi.org/10.1038/s41422-019-0185-0
}

\begin{abstract}
Regulation of gene expression is a major component of insulin action, which is classically thought to occur via phosphorylation, relocalization and/or processing of transcriptional regulators downstream of the insulin receptor (IR) signaling cascade. A recent study in Cell suggests a mechanism by which IR itself translocates to the nucleus and forms transcriptional complexes with RNA polymerase II, host cell factor-1 and transcription factors to directly regulate gene expression, thus adding a new complementary pathway to control this important set of insulin actions.
\end{abstract}

Insulin is the central regulator of metabolism. Insulin controls the balance between fuel utilization and storage, thus maintaining glucose levels during cycles of feeding and fasting. These functions occur in part through effects of insulin to regulate gene expression in a tissue- and cell-specific manner. ${ }^{1}$ At the molecular level, insulin binds to insulin receptors (IR) present on cell surface activating IR tyrosine kinase activity. This initiates a broad network of protein phosphorylation, including phosphorylation of IRS-1 leading to activation of the phosphatidylinositol 3-kinase (PI3K)/ AKT pathway involved in most metabolic actions of insulin and phosphorylation of SHC with activation of the RAS/MAP kinase pathway that mediates effects on growth and proliferation. ${ }^{2}$ Each of these pathways results in changes in phosphorylation, processing and expression of downstream transcriptional regulators, forming the widely accepted model to explain the effects of insulin to regulate gene expression (Fig. 1, left). The most wellstudied example is the forkhead family box O (FOXO) transcription factors which, upon insulin stimulation, are phosphorylated by AKT, bind to $14-3-3$ proteins and are thus excluded from the nucleus, limiting their ability to regulate gene expression. ${ }^{3}$ Insulin also promotes phosphorylation of FOXK transcription factors ${ }^{4}$ and phosphorylation and processing of SREBP- $1 c^{5}$ but in these cases, this promotes migration of the FOXKs and SREBP1c into the nucleus where they can regulate transcription.

In a recent paper in Cell, Hancock et al. ${ }^{6}$ propose a new model of insulin regulation of transcription in which IR itself translocates to the nucleus, where it interacts with RNA polymerase II (Pol II) on gene promoters and induces transcription (Fig. 1, right). IR was identified in the nucleus by binding studies over 40 years ago and suggested to play a role in mRNA export, ${ }^{7,8}$ but over the years, interest in a potential role of nuclear IR was lost, and the functional significance of these observations remained elusive. In this new report, mass spectrometry analysis of immunoprecipitated IR from mouse livers revealed an unexpected association of the receptor with multiple subunits of the Pol II complex. ChIP-seq analysis of IR in hepatocytes reveal that $73 \%$ of genomic regions where IR peaks were detected overlapped with Pol II occupancy, predominantly on actively transcribed promoter regions. Insulin stimulation led to increased nuclear IR and binding of IR on chromatin. These IRbound promoters were highly enriched for genes known to be involved in insulin functions in liver, including lipid metabolism, protein synthesis and transcription, as well as for disease pathways where IR signaling is typically dysfunctional such as diabetes and Alzheimer's disease. On the other hand, these sites did not include genes involved in pathways of glucose metabolism, such as gluconeogenesis and glycogen synthesis, which are major metabolic functions of insulin in liver.

Through a combination of motif analysis and mass spectrometry, Hancock et al. ${ }^{6}$ found that the transcriptional coregulator host cell factor-1 (HCF-1) is required for the assembly of IR transcriptional complexes, on at least a subset of genes. Since HCF-1 lacks direct DNA-binding activity, an important step moving forward will be to determine the DNA-binding proteins and transcription factors that mediate IR-HCF-1-Pol II complex formation, how these are regulated and what genes they regulate. Hancock et al. $^{6}$ suggest that one potential transcription factor mediating IR binding to gene promoters is THAP11, a gene involved in mouse embryonic development, but not previously associated with insulin action. Another possibility may be the FOXK transcription factors, since a recent study by Sakaguchi et al. ${ }^{4}$ showed that FOXK1 can physically interact with IR and translocate to the nucleus in response to insulin where it controls genes involved in mitochondrial metabolism and cell cycle. Clearly, additional work will be needed to provide a full picture of the link between nuclear IR and DNA-binding factors in the direct regulation of gene expression.

The new model proposed by Hancock et al. expands the vision of how insulin can act to regulate gene expression indicating at least three complementary mechanisms. Signaling molecules downstream of IR, such as AKT, GSK3 $\beta$ and mTOR, can directly phosphorylate transcription factors or their coregulators promoting their movement into or out of the nucleus (FOXKs vs FOXOs); insulin can promote the processing or expression, with or without phosphorylation, of transcription factors (SREBP1C and CHREBP); and IR can translocate to the nucleus where it directly interacts with the transcriptional machinery to modulate its activity. This multiplicity of pathways expands the typical outside-to-inside view of signaling cascades and provides new insights into their wiring. Moving forward, it will be important to address the mechanism by which IR translocates to the nucleus, as well as the molecular actions of IR inside the nucleus. Endocytosis-mediated receptor internalization is a well-described phenomenon in control or recycling and degradation of IR and other receptor kinases. ${ }^{9}$ Hancock et al. ${ }^{6}$ and others have shown an interaction between IR and clathrin heavy chain $(\mathrm{CLH})$, a major component of the endocytosis

${ }^{1}$ Section of Integrative Physiology and Metabolism, Joslin Diabetes Center, Brigham and Women's Hospital Department of Internal Medicine, Harvard Medical School, Boston, MA 02215, USA

Correspondence: C. Ronald Kahn (c.ronald.kahn@joslin.harvard.edu)

Published online: 3 June 2019 


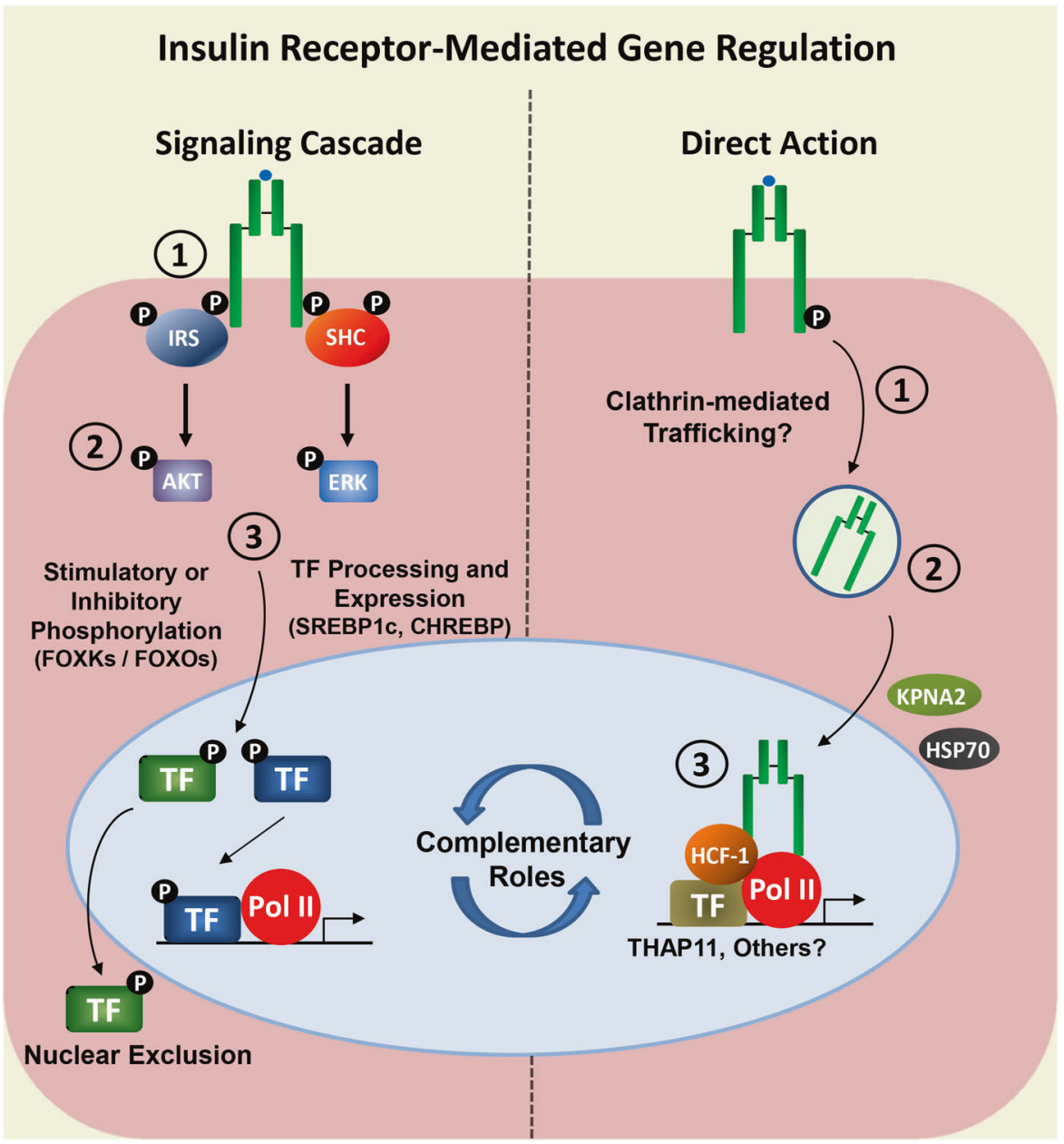

Fig. 1 Left, insulin receptor (IR) signaling cascade-mediated gene regulation. (1) Activated IR recruits and phosphorylates substrate proteins such as IRS and SHC; (2) IRS phosphorylation leads to PI3K-dependent AKT activation. SHC phosphorylation leads to RAS-dependent ERK activation; (3) Activated downstream signaling kinases phosphorylate transcription factors (TFs) and promote changes in TF nuclear translocation, post-translational processing and/or expression. Stimulatory phosphorylation promotes transcriptional complex formation leading to increased transcription. Inhibitory phosphorylation promotes nuclear exclusion and inaccessibility to chromatin leading to decreased transcription. Right, direct IR action on gene regulation. (1) Activated IR on the cell surface undergo internalization process through pathways involving clathrin-coated pits. (2) IR translocates to the nucleus. Mass spectrometry data suggests involvement of the importin family of proteins represented by karyopherin alpha 2 (KPNA2), as well as heat shock protein 70 (HSP70). (3) Nuclear IR associates with chromatin and forms a complex containing HCF-1, Pol II and DNA-binding transcription factors, such as THAP11 and potentially others

machinery, raising the possibility that endocytosis may regulate IR transport to the nucleus. Additionally, although this paper identifies the presence of IR in transcriptional complexes, the full functional significance remains unclear. Neither HCF-1 nor THAP11 have previously been implicated in insulin action on gene expression in mammalian cells. While knocking down HCF1 impaired IR regulation of fatty acid synthase (a genetic region associated with IR binding), it did not impair insulin regulation of IGFBP1, a gene downstream of the PI3K-AKT pathway (where IR binding was not detected), suggesting dual functionality of IR to directly and indirectly mediate transcriptional events. In the future, it will be important to understand the interplay between these pathways and how they might complement each other to regulate gene expression (Fig. 1).

An even more important question is the contribution of this pathway to normal physiology and disease. Gene expression profiling of tissue biopsies from type 2 diabetic subjects has consistently shown changes in gene expression, ${ }^{10,11}$ but the molecular pathways underlying these effects are still poorly defined. Pointing in this direction, Hancock et al. ${ }^{6}$ show that IR association with chromatin is reduced in livers of genetically obese $o b / o b$ mice suggesting that nuclear IR functions may be modified in this insulin resistant state, although the downregulation of membrane $I R$ which occurs in obesity likely also contributes to this effect. Moving forward, it will be crucial to address to what extent direct actions of IR in the nucleus play a role in normal physiology of feeding and fasting, and whether this is modified in other models of diabetes and insulin resistance, including human obesity and type 1 or 2 diabetes. As we begin to understand the significance of pioneer observations from over four decades ago, it will also be exciting to determine whether other membrane receptors can act like the IR and take gene regulation into their own hands by going nuclear.

\section{REFERENCES}

1. Batista, T. M. et al. Cell Rep. 26, 3429-3443 (2019).

2. Boucher, J., Kleinridders, A. \& Kahn, C. R. Cold. Spring. Harb. Perspect. Biol. 6, https://doi.org/10.1101/cshperspect.a009191 (2014). 
3. Nakae, J., Barr, V. \& Accili, D. EMBO J. 19, 989-996 (2000)

4. Sakaguchi, M. et al. Nat. Commun. 10, 1582 (2019).

5. Shimano, H. \& Sato, R. Nat. Rev. Endocrinol. 13, 710-730 (2017).

6. Hancock, M. L. et al. Cell 177, 722-736 (2019).

7. Goldfine, I. D. \& Smith, G. J. Proc. Natl. Acad. Sci. USA 73, 1427-1431 (1976).
8. Purrello, F., Burnham, D. B. \& Goldfine, I. D. Proc. Natl. Acad. Sci. USA 80, 1189-1193 (1983).

9. Goh, L. K. \& Sorkin, A. Cold. Spring. Harb. Perspect. Biol. 5, a017459 (2013).

10. Mootha, V. K. et al. Nat. Genet. 34, 267-273 (2003)

11. Patti, M. E. et al. Proc. Natl Acad. Sci. USA 100, 8466-8471 (2003). 